\title{
Schader-Preis 2014 für Stephan Leibfried
}

Professor Dr. Stephan Leibfried (Bremen) erhält den Schader-Preis 2014. Mit dem Preis zeichnet die Schader-Stiftung Gesellschaftswissenschaftlerinnen und -wissenschaftler aus, die durch ihre wissenschaftliche Arbeit und ihr öffentliches Wirken wichtige Beiträge für die Lösung gesellschaftlicher Probleme geleistet haben.

Stephan Leibfried erhält den Preis in Anerkennung seiner international herausragenden und auch praktisch ambitionierten Verdienste um die Entwicklung der sozialwissenschaftlichen Forschung zum Sozialstaat und zur allgemeinen jüngeren Staatsentwicklung. Seiner Initiative und Energie ist im Wesentlichen die Gründung des ,Zentrums für Sozialpolitik“ (ZeS) 1988 an der Universität Bremen zu verdanken. Das ZeS ist mittlerweile $\mathrm{zu}$ einer international führenden Forschungseinrichtung zur Sozialpolitik und vergleichenden Wohlfahrtsstaatsforschung geworden. Zahlreiche Forschungsinitiativen - u. a. der soziologisch geprägte Sonderforschungsbereich „Statuspassagen und Risikolagen im Lebensverlauf" (1988-2001) und der politikwissenschaftliche Sonderforschungsbereich zur „Staatlichkeit im Wandel“ (2003-2014) - sind maßgeblich von Stephan Leibfried in Gang gesetzt und mit Leben erfüllt worden. Seit 2008 engagiert sich Stephan Leibfried auch in wissenschaftspolitischen Debatten, insbesondere durch kritisch-konstruktive Kommentare zur Exzellenzinitiative des Bundes und der Länder und zu ihrer Weiterführung.

Stephan Leibfried ist Initiator und Mitherausgeber des „Oxford Handbook of the Welfare State“, das den internationalen Forschungsstand systematisch zusammenfasst und einen großen Widerhall gefunden hat. Derzeit arbeitet er hauptverantwortlich an der Herausgabe des „Oxford Handbook of Transformations of the State“, das 2014/2015 erscheinen wird.

Der Schader-Preis wird jährlich von der Schader-Stiftung verliehen und ist mit $15.000 €$ dotiert. Die Jury bildet der Senat der Stiftung. Zu den bisherigen Preisträgern zählen unter anderem Jutta Allmendinger (2013), Paul Kirchhof (2012), Jan Philipp Reemtsma (2011), Wolf Lepenies (2010), Lord Ralf Dahrendorf (2009), Klaus von Beyme (2008), FranzXaver Kaufmann (2007), Gesine Schwan (2006) und Ulrich Beck (2005). Die SchaderStiftung fördert die Gesellschaftswissenschaften und deren Dialog mit der Praxis.

(C) Springer Fachmedien Wiesbaden 2014 